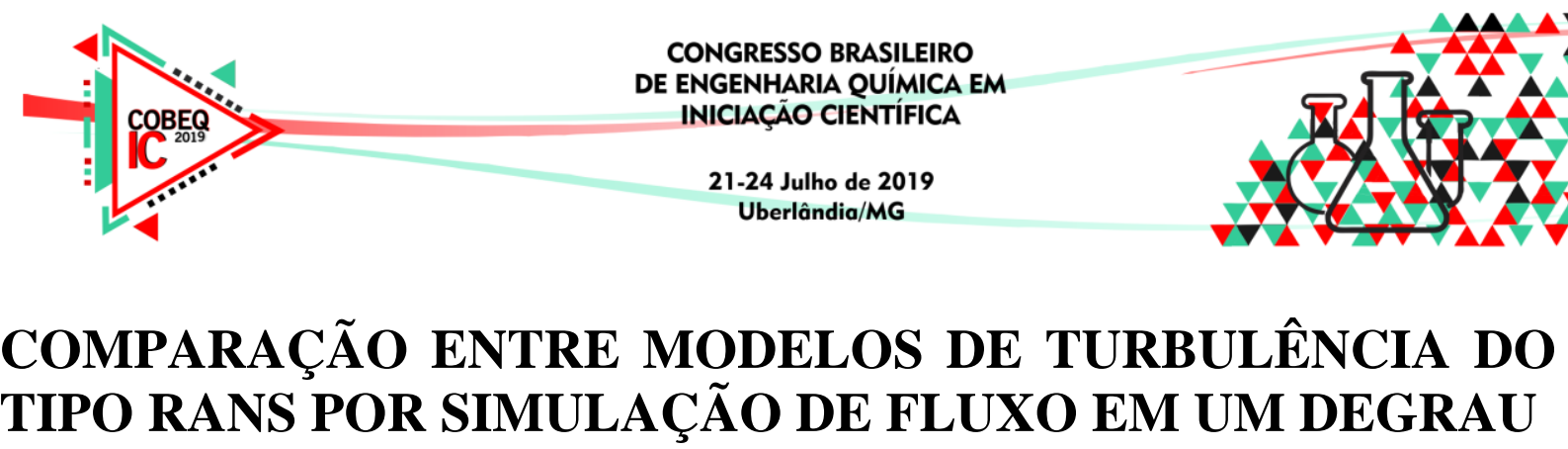

\author{
M. A. R. SILVA ${ }^{1}$, G. B. CORREA ${ }^{1}$ e J. F. MITRE ${ }^{1}$ \\ ${ }^{1}$ Universidade Federal Fluminense, Departamento de Engenharia Química e de Petróleo \\ E-mail para contato:jf_mitre@id.uff.br
}

\begin{abstract}
RESUMO - Escoamentos turbulentos são muito comuns da indústria, porém sua modelagem ainda produz muitos questionamentos. A modelagem mais rigorosa envolve a simulação direta das equações de Navier-Stokes, porém, essa é inviável na maior parte das aplicações práticas. Várias modelagens propostas simplificadas surgiram ao longo dos anos, entretanto, a acurácia desses modelos é continuamente testada na literatura. Esse trabalho avalia os modelos tipo RANS disponíveis no OpenFOAM em um escoamento em expansão devido à presença de um degrau e compara seus resultados com dados experimentais e simulações DNS da literatura. Nos resultados, pode-se perceber que a variação do coeficiente de pressão ao longo da distância percorrida e a distribuição da velocidade em seções transversais específicas estão bem concordantes entre si e com a literatura.
\end{abstract}

\title{
1. INTRODUÇÃO
}

Simulações numéricas envolvendo escoamentos turbulentos configuram grande desafio em fluidodinâmica computacional, principalmente em número de Reynolds elevado. A abordagem de problemas típicos desta natureza consiste em modelagem por meio de equações de conservação seguida de resolução através de alguns métodos numéricos e algoritmos acoplados a diversos tipos de modelos de turbulência (DING et al., 2010).

O cálculo direto de todas as escalas de tempo e comprimento do escoamento requer o uso de malhas extremamente refinadas em simulação denominada Direct Numerical Simulation (DNS), que é pouco aplicada em casos práticos com alto número de Reynolds. A modelagem mais utilizada é baseada na equação média de Navier Stokes (Reynolds-averaged Navier Stokes equations, RANS), cujas soluções envolvem a decomposição das escalas em um campo médio no tempo e um campo de flutuações. Assim, é possível deduzir equações de conservação médias, que resultam no tensor de Reynolds (MOUKALLED et al., 2016).

A modelagem RANS envolve duas classes de modelos: hipótese de Boussinesq e fechamento de segunda ordem. Com relação à hipótese de Boussinesq, o tensor de Reynolds é modelado considerando que há uma dependência com uma viscosidade turbulenta e um tensor deformação. A viscosidade turbulenta, por sua vez, pode ser obtida a partir de diversos modelos. Os modelos de fechamento de segunda ordem configuram a melhor opção para escoamentos com comportamento anisotrópico, apesar do maior custo computacional. Tais modelos são uma alternativa ao fechamento das equações RANS por meio da formulação de equações de transporte diretas para o tensor de Reynolds (BLAZEK, 2001).

Um problema típico e amplamente empregado na verificação da qualidade de modelos de turbulência é a expansão de um fluido pela presença de um degrau em um trecho do escoamento. 


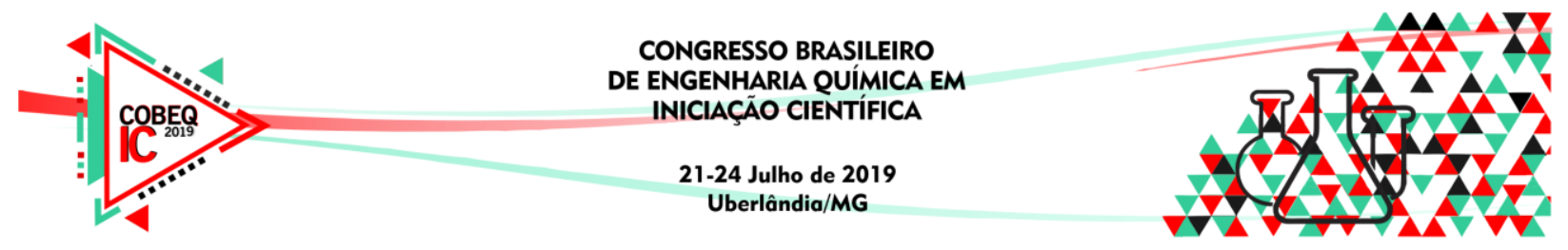

Em vários trabalhos (ROBERTSON et al., 2015; DING et al., 2012; HUNGLE et al., 1997), tem sido mostrado problemas deste tipo para variados modelos de turbulência e números de Reynolds.

Dentre os softwares que permitem simulações envolvendo turbulência, o OpenFOAM (OpenFOAM, 2018) configura uma importante ferramenta por se tratar de um programa gratuito e opensource. No interior de sua estrutura, há vários solvers (aplicativos), que são projetados para resolver um problema específico em mecânica do fluido (ou contínuo), e utilitários, projetados para executar tarefas que envolvem manipulação de dados.

O presente trabalho objetiva a avaliação da eficiência de modelos de turbulência do tipo RANS disponíveis nas bibliotecas padrões do OpenFOAM por meio do escoamento em um degrau. Para tal, uma comparação entre os resultados numéricos e experimentais foi realizada. Concomitantemente, a estabilidade para diferentes malhas foi abordada.

\section{DESCRIÇÃO DAS CONDIÇÕES DO PROBLEMA}

A Figura 1 é uma representação esquemática do domínio computacional 2D empregado nas simulações. Considera-se que um fluido newtoniano está escoando através de um túnel retangular e, a partir de determinado ponto, a presença de um degrau promove alterações no comportamento do fluxo. Tem-se que a altura do degrau $(\mathrm{H})$ apresenta um valor de $0,0381 \mathrm{~m}$, enquanto a proporção $\mathrm{H}: \mathrm{H}_{1}$ corresponde a $1: 2$. O comprimento total a jusante $(\mathrm{L}) \mathrm{e}$ a montante $\left(\mathrm{L}_{1}\right)$ do degrau são de, respectivamente, $0,80 \mathrm{~m}$ e $0,32 \mathrm{~m}$. Na Figura 1, também é possível visualizar as seções transversais $\mathrm{x} / \mathrm{H}=8,00$ e $\mathrm{x} / \mathrm{H}=5,33$, representadas em vermelho, as quais serão abordadas nos resultados apresentados.

Figura 1 - Esquema do domínio computacional associado a suas dimensões e contornos.

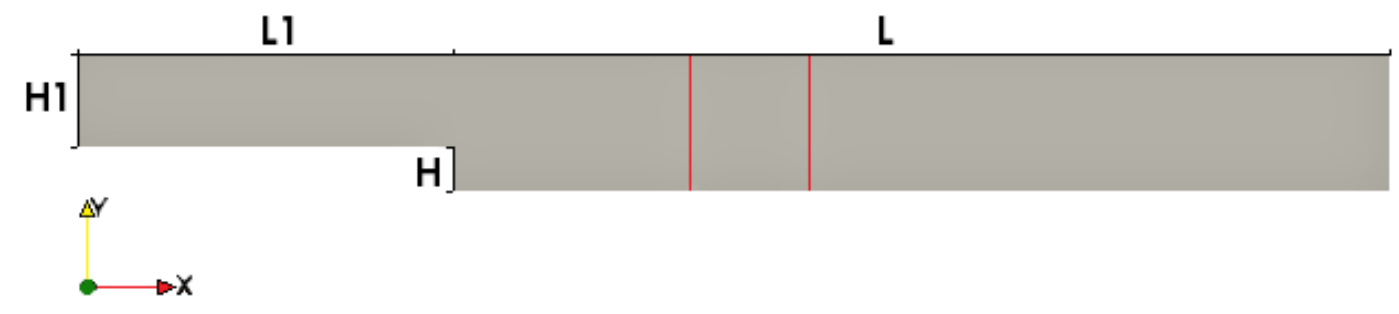

Uma velocidade inicial ( $\left.\mathrm{U}_{0}\right)$ de $18,2 \mathrm{~m} / \mathrm{s}$ na direção $\mathrm{x}$ foi imposta como condição de contorno na entrada. $\mathrm{O}$ número de Reynolds foi definido com base na velocidade $\mathrm{U}_{0}$ e altura $\mathrm{H}$, apresentando valor de 44000. A velocidade na parede de cima e debaixo apresenta condição de não deslizamento e, na saída, é dada por gradiente nulo. Para a pressão relativa, a saída é representada por um valor nulo e a entrada, assim como paredes de cima e debaixo, por gradiente nulo.

As equações básicas que governam o problema são a equação média da continuidade e a equação média de conservação de quantidade de movimento linear (OpenFOAM, 2018). As equações foram resolvidas por meio do método dos volumes finitos, com auxílio do OpenFOAM, em 4 diferentes tipos de malhas ortogonais, com número total de células de, respectivamente, 52200, 128000, 201600 e 263200. Tais malhas foram produzidas com o utilitário blockMesh e possibilitaram a realização do teste de independência de malha. 


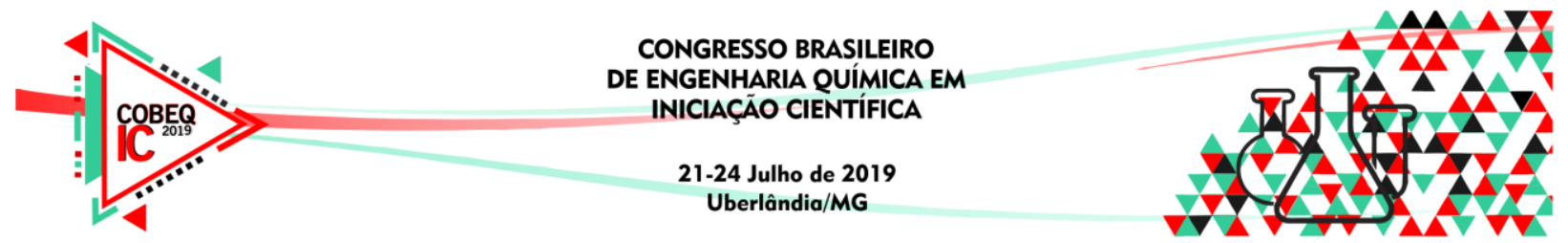

No que tange aos esquemas numéricos empregados, tem-se que foram escolhidos visando uma maior estabilidade e acurácia. Foram utilizados, primeiramente, métodos robustos de primeira ordem e, posteriormente, de acordo com o andamento da simulação, métodos de segunda ordem.

Com relação aos métodos numéricos empregados para resolução dos sistemas lineares, utilizou-se o método dos gradientes conjugados PCG com DIC, para matrizes simétricas, e o método dos gradientes biconjugados PBiCG com DILU, para solução das matrizes assimétricas. Além disso, escolheu-se um solver baseado no algoritmo SIMPLE, o qual configura um método semi-implícito que acopla pressão e velocidade para fluidos incompressíveis.

$\mathrm{Na}$ aplicação da hipótese de Boussinesq, os modelos padrões $\kappa-\varepsilon$ e $\kappa-\omega$, além de variantes, têm suas equações bases disponibilizadas por Robertson et al. (2015). Segundo Moukalled et al. (2016), as derivações dos modelos $\kappa-\varepsilon$ padrão, Realizable e RNG são mais adequadas para casos totalmente turbulentos com baixa previsibilidade próximo à parede. $\mathrm{A}$ modelagem de fluxos próximos a paredes sólidas requer as correções para efeitos de baixo número de Reynolds, o que é possível por meio de algumas variantes dos modelos $\kappa-\varepsilon$. Nestes casos, é feito um amortecimento da viscosidade turbulenta perto da parede através da utilização de uma função específica. Outra possibilidade para modelagem na parede é a utilização de funções de parede, que são derivadas empiricamente. Nestes casos, o parâmetro $\mathrm{y}^{+}$(distância de parede adimensional) se torna fundamental para julgar a aplicabilidade do método.

\section{ANÁLISE DOS RESULTADOS}

Antes da simulação ser iniciada, verificaram-se erros topológicos e dados importantes das malhas geradas. Tal procedimento é fundamental, pois malhas ruins geram oscilações numéricas, diminuindo a acurácia numérica da solução. Foram bem refinadas as regiões de alto gradiente das variáveis, a fim de evitar difusão numérica, e a entrada, para captura do desenvolvimento da camada limite. Tem-se que a convergência de malha foi realizada para todos os modelos, podendo ser exemplificada para $0 \kappa-\varepsilon$ padrão por meio da Figura 2. Para as malhas 1, 2 e 3, os resultados obtidos desse e dos outros modelos são praticamente idênticos.

Figura 2 - Distribuição de velocidade horizontal média na seção transversal x/H=8,00 para diferentes malhas do modelo $\kappa-\varepsilon$ padrão.

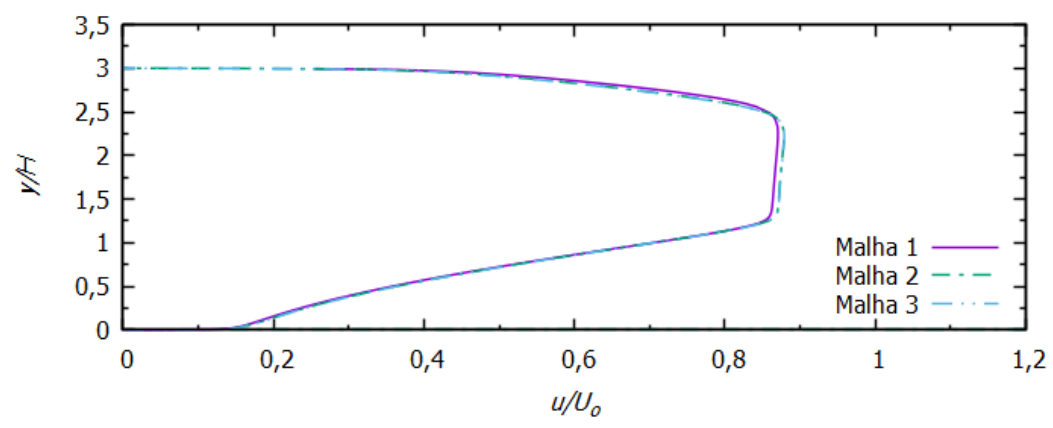

Na Figura 2, observa-se que o desvio entre as malhas 2 e 3 é menor do que entre as malhas 1 e 2, mostrando uma diferença não significativa entre os refinamentos. A malha escolhida foi a mais refinada (malha 3), já que é a que apresenta maior acurácia. Na Tabela 1, é possível obter os valores de $\mathrm{y}^{+}$, em relação a parede inferior, das malhas finais escolhidas para representação dos modelos de turbulência utilizados. 


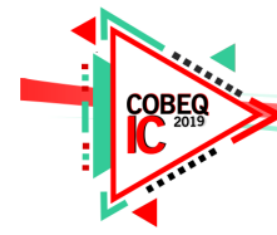

Considerando que funções de parede foram utilizadas para os modelos, por análise da Tabela 1, observa-se que $\mathrm{o}^{+}$das malhas dos modelos $\kappa-\varepsilon$ se encontram refinadas excessivamente, já que o recomendado varia entre $30<\mathrm{y}^{+}<100$. No entanto, este foi um modo de assegurar maior acurácia aos métodos. Para modelos $\kappa-\omega$, valores de $\mathrm{y}^{+}$próximos de 1 foram estabelecidos.

Tabela $1-\mathrm{y}^{+}$para os modelos de turbulência utilizados.

\begin{tabular}{|c|c|c|c|c|}
\hline Modelo & Malha & $\mathrm{y}^{+}$mínimo & $\mathrm{y}^{+}$médio & $\mathrm{y}^{+}$máximo \\
\hline \hline$\kappa-\varepsilon$ & 3 & 0,12 & 2,97 & 6,39 \\
\hline$\kappa-\varepsilon$ RNG & 3 & $6,94.10^{-2}$ & 2,79 & 6,32 \\
\hline$\kappa-\omega$ & 4 & $7,46.10^{-6}$ & 0,21 & 1,52 \\
\hline$\kappa-\omega$ SST & 3 & $6,81.10^{-6}$ & 0,25 & 1,46 \\
\hline Realizable $\kappa-\varepsilon$ & 1 & $3,91.10^{-2}$ & 4,70 & 9,82 \\
\hline Lam-Bremhorst $\kappa-\varepsilon$ & 3 & $2,00.10^{-2}$ & 1,69 & 3,85 \\
\hline Launder-Sharma $\kappa-\varepsilon$ & 3 & $1,76.10^{-3}$ & 1,49 & 3,77 \\
\hline
\end{tabular}

A Figura 3 fornece dados a respeito do coeficiente de pressão $\left(C_{p}\right)$ ao longo da superfície da parede inferior, sendo a sua formulação dada pela Equação 1. Foi feita uma comparação com dados experimentais de Kim et al. (1980) e resultados de simulação DNS de Ding et al. (2012) baseados nestes dados. Observa-se uma boa concordância dos resultados apresentados.

$$
C_{p}=2 \frac{\left(p-p_{0}\right)}{\rho U_{0}^{2}}
$$

em que p é a pressão relativa, $p_{0}$ é a pressão média na seção transversal de entrada e $\rho$ é a massa específica.

Figura 3 - Distribuição do coeficiente de pressão ao longo do escoamento para diferentes modelos de turbulência.

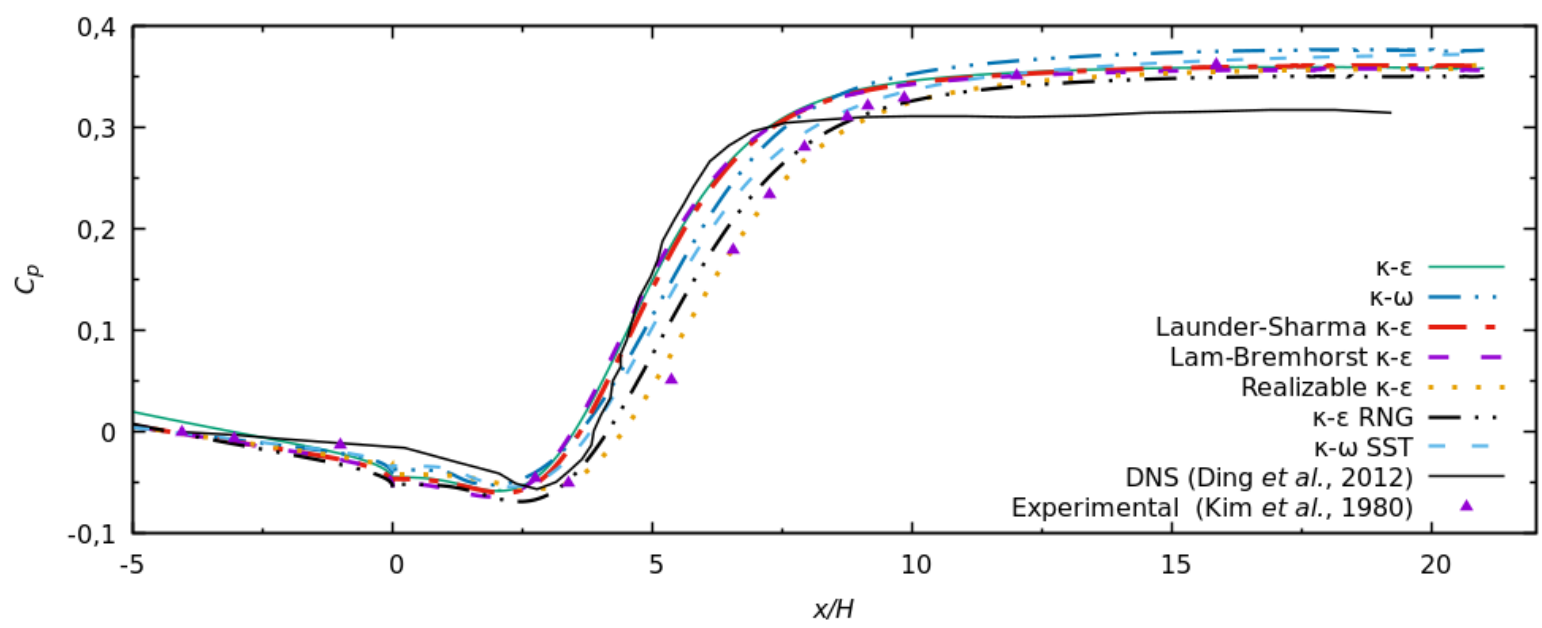

As Figuras 4 e 5 fornecem os resultados da velocidade média para as seções transversais localizadas em $\mathrm{x} / \mathrm{H}=5,33$ e $\mathrm{x} / \mathrm{H}=8,00$, respectivamente. Dados experimentais de Kim et al. (1980) e resultados de simulação DNS obtidos por Ding et al. (2012) também são apresentados.

Figura 4 - Distribuição de velocidade horizontal média na seção transversal x/H=5,33. 

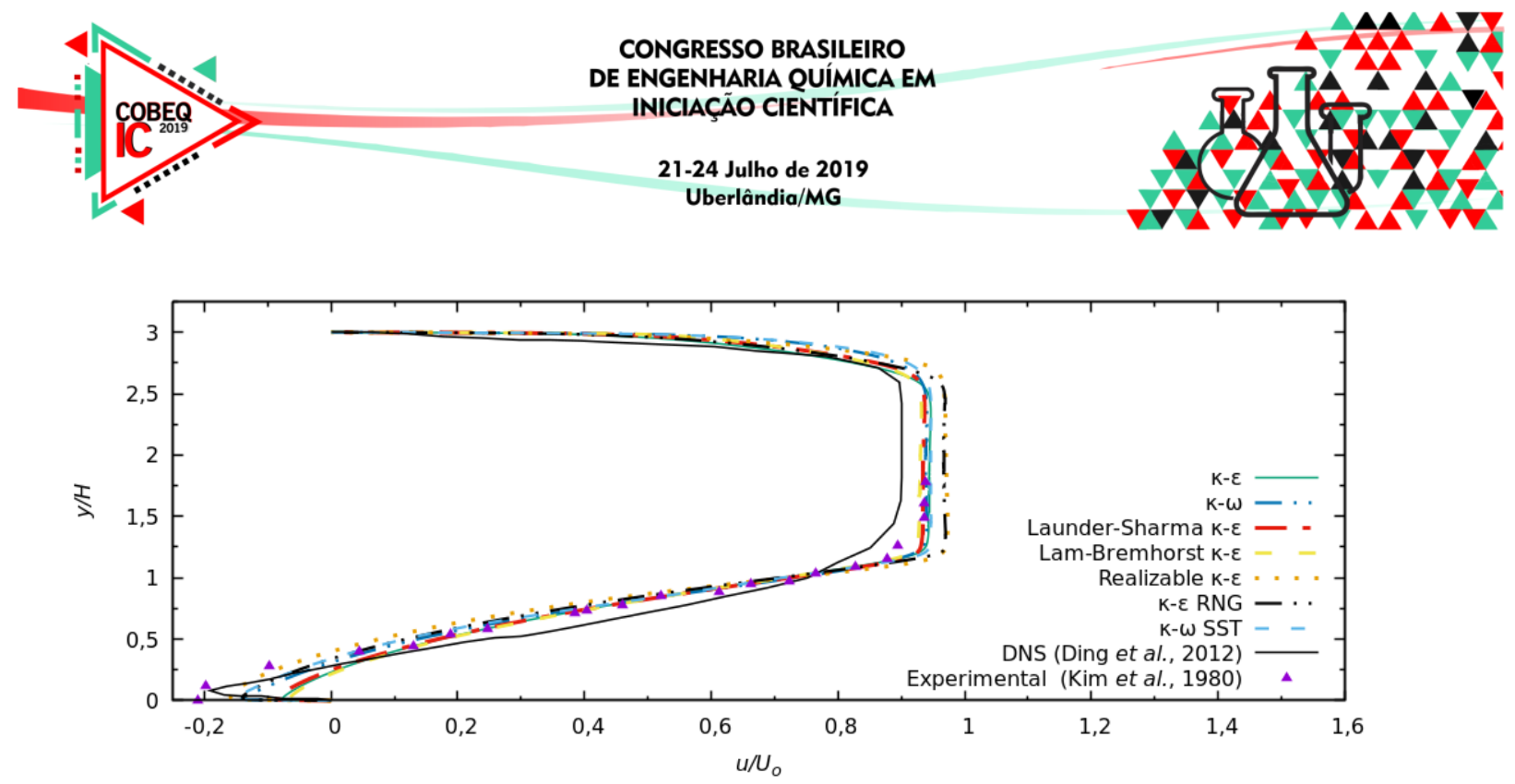

Figura 5 - Distribuição de velocidade horizontal média na seção transversal x/H=8,00.

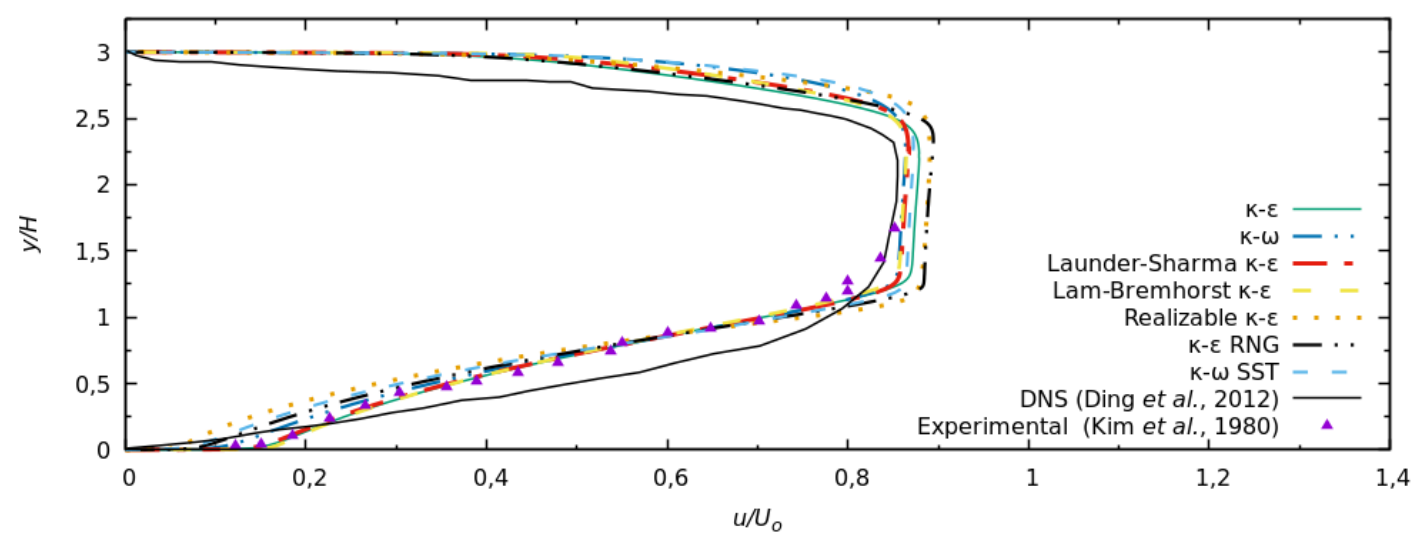

Conforme observado nas Figuras 4 e 5, os modelos $\kappa-\varepsilon$, Launder-Sharma $\kappa-\varepsilon$ e LamBremhorst $\kappa-\varepsilon$ apresentaram uma boa concordância entre si, assim como os modelos RNG $\kappa$ $\varepsilon$ e Realizable $\kappa-\varepsilon$ e os modelos $\kappa-\omega$ e $\kappa-\omega$ SST.

Em $\mathrm{x} / \mathrm{H}=5,33$, comparando a proximidade dos valores calculados com os experimentais, próximo à parede inferior, os modelos Realizable e RNG foram os mais satisfatórios, embora não tenham sido os melhores para o seio do fluido. $\kappa-\varepsilon$, Launder-Sharma $\kappa-\varepsilon$ e Lam-Bremhorst $\kappa-\varepsilon$ descreveram bem o seio do fluido e os modelos $\kappa-\omega$ e $\kappa-\omega$ SST foram os mais satisfatórios em escala geral. Já para $\mathrm{x} / \mathrm{H}=8,00, \kappa-\varepsilon$, Launder-Sharma $\kappa-\varepsilon$ e Lam-Bremhorst $\kappa-\varepsilon$ têm uma boa concordância com a parede e seio do fluido, os modelos RNG e Realizable são os menos satisfatórios e os modelos $\kappa-\omega$ apresentam ajuste intermediário.

Os resultados apresentados anteriormente são previsíveis, considerando as condições nas quais cada modelo é mais adequado. Como, no OpenFOAM, funções de parede foram especificadas para todos os modelos, justifica-se o fato de o modelo $\kappa-\varepsilon$ padrão ser próximo ao Launder-Sharma $\kappa-\varepsilon$ e Lam-Bremhorst $\kappa-\varepsilon$, que são modelos para low Reynolds.

Os modelos RNG e Realizable, embora sejam do tipo high Reynolds, tiveram um desvio maior na região do seio do fluido, provavelmente devido ao fato de o fluido estar confinado, implicando em alterações adicionais. Estes modelos apresentam uma forma modificada da equação do $\varepsilon$, de forma a tentarem explicar as diferentes escalas de movimento. Para o modelo $\kappa$ - $\omega$ SST, a sua concordância satisfatória se deve ao fato de configurar uma associação entre 


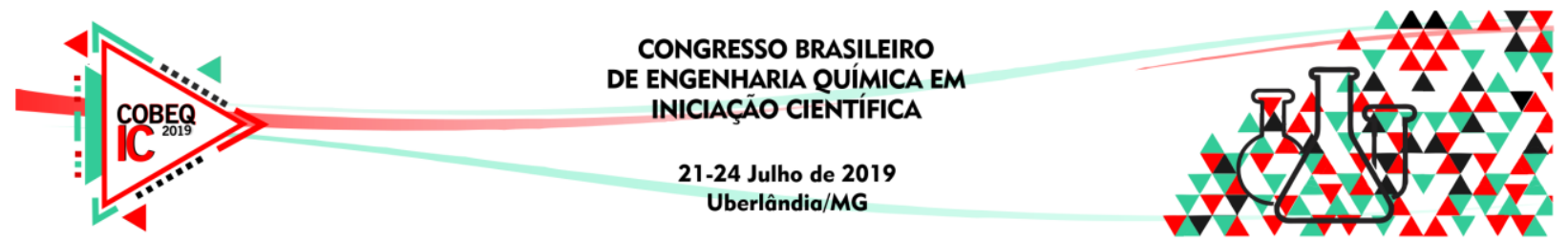

os modelo $\kappa-\varepsilon$, que tem boa previsibilidade no seio do fluido, e $\kappa-\omega$, que tem boa previsibilidade próximo à parede.

Os modelos utilizados neste trabalho são semi-empíricos e, portanto, resultam em valores diferentes dos dados testados. Os erros observados, além do modelo em si, também são decorrentes dos métodos numéricos utilizados, condições de contorno e condições iniciais. Apesar destes fatores, a concordância geral dos modelos foi satisfatória.

\section{CONCLUSÕES}

Após implementação de 7 modelos de turbulência do tipo RANS, tem-se que, dentre todos os analisados, os do tipo $\kappa-\varepsilon$, em geral, descreveram melhor o escoamento. Como exceções, os modelos Realizable e RNG possuiram concordância com os dados experimentais somente em $\mathrm{x} / \mathrm{H}=5,33$ e apenas próximo a parede e os modelos $\kappa-\varepsilon$, Launder-Sharma e LamBremhorst só discordaram dos dados experimentais na parede em $\mathrm{x} / \mathrm{H}=5,33$. Já para os modelos $\kappa$ - $\omega$, uma boa concordância em $\mathrm{x} / \mathrm{H}=5,33$ foi encontrada, porém, com menor acurácia em $\mathrm{x} / \mathrm{H}=8,00$. Nota-se que, mesmo com um grande número de volumes, não foi possivel obter $\mathrm{y}^{+}<1$ para os modelos $\kappa-\omega$, o que se deve ao fato de a malha escolhida ser maior do que o necessário, implicando em desperdício de malha. Entretanto, não houve perda de qualidade da solução nem a necessidade de $30<\mathrm{y}^{+}<100$ para os modelos $\kappa-\varepsilon$.

\section{REFERÊNCIAS}

BLAZEK, J. Computational Fluid Dynamics: Principles and Applications. Oxford: ELSEVIER SCIENCE Ltd, 2001.

DING, D. Y.; WU, S. Direct numerical simulation of turbulent flow over backward-facing at high Reynolds numbers. Sci. China Tech. Sci., v. 55, p. 3213-3222, 2012.

HUNGLE, L.; PARVIZ, M.; JOHN, K. Direct numerical simulation of turbulent flow over a backward-facing step. J. Fluid Mech., v. 330, p. 349-374, 1997.

KIM, J.; KLINE, S. L.; JOHNSTON, J. P. Investigation of a Reattaching Turbulent Shear Layer: Flow Over a Backward-Facing Step. Journal of Fluids Engineering, v. 102, p. 302 $-308,1980$.

MOUKALLED, F.; MANGANI, L.; DARWISH, M. The Finite Volume Method in Computational Fluid Dynamics: An Advanced Introduction with OpenFOAM and Matlab. New York: Springer, 2016.

OpenFOAM. The Open Source CFD Toolbox, version 6.0.x. London, UK: The OpenFOAM Foundation Ltd, 2018.

ROBERTSON, E.; CHOUDHURY, V.; BHUSHAN, S.; WALTERS, D.K. Validation of OpenFOAM numerical methods and turbulence models for incompressible bluff body flows. Computers and Fluids, v. 123, p. 122-145, 2015. 